\title{
Comparison and functional implications of the 3D architectures of viral tRNA-like structures
}

\author{
JOHN A. HAMMOND, ${ }^{1}$ ROBERT P. RAMBO, ${ }^{2}$ MEGAN E. FILBIN, ${ }^{1}$ and JEFFREY S. KIEFT ${ }^{\mathbf{1}}$ \\ ${ }^{1}$ Department of Biochemistry and Molecular Genetics, University of Colorado Denver School of Medicine, Aurora, Colorado 80045, USA \\ ${ }^{2}$ Life Science Division, Lawrence Berkeley National Laboratory, Berkeley, California 94720, USA
}

\begin{abstract}
RNA viruses co-opt the host cell's biological machinery, and their infection strategies often depend on specific structures in the viral genomic RNA. Examples are tRNA-like structures (TLSs), found at the $3^{\prime}$ end of certain plant viral RNAs, which can use the cell's aminoacyl tRNA-synthetases (AARSs) to drive addition of an amino acid to the $3^{\prime}$ end of the viral RNA. TLSs are multifunctional RNAs involved in processes such as viral replication, translation, and viral RNA stability; these functions depend on their fold. Experimental result-based structural models of TLSs have been published. In this study, we further examine these structures using a combination of biophysical and biochemical approaches to explore the three-dimensional (3D) architectures of TLSs from the turnip yellow mosaic virus (TYMV), tobacco mosaic virus (TMV), and brome mosaic virus (BMV). We find that despite similar function, these RNAs are biophysically diverse: the TYMV TLS adopts a characteristic tRNA-like L shape, the BMV TLS has a large compact globular domain with several helical extensions, and the TMV TLS aggregates in solution. Both the TYMV and BMV TLS RNAs adopt structures with tight backbone packing and also with dynamic structural elements, suggesting complexities and subtleties that cannot be explained by simple tRNA mimicry. These results confirm some aspects of existing models and also indicate how these models can be improved. The biophysical characteristics of these TLSs show how these multifunctional RNAs might regulate various viral processes, including negative strand synthesis, and also allow comparison with other structured RNAs.
\end{abstract}

Keywords: probing/RNA structure; small-angle X-ray scattering (SAXS); tRNA-like structure (TLS); viral RNA

\section{INTRODUCTION}

Transfer RNAs (tRNAs) physically link a messenger RNA's (mRNA's) sequence and the correct amino acids and are involved in processes such as transcriptional regulation (Grundy and Henkin 1993), viral packaging (Annamalai and Rao 2007), amino acid biosynthesis (Sheppard et al. 2008), and viral genome replication (Wain-Hobson et al. 1985). Like many biologically important RNAs, the roles of tRNA depend on its folded structure; in the case of tRNA, this is the classic "L-shaped" fold (Robertus et al. 1974; Suddath et al. 1974). The tRNA fold is a versatile scaffold that is mimicked in whole or in part by many other RNAs and proteins (Tsonis and Dwivedi 2008).

Reprint requests to: Jeffrey S. Kieft, Department of Biochemistry and Molecular Genetics, University of Colorado Denver School of Medicine, Mail Stop 8101, P.O. Box 6511, Aurora, CO 80045, USA; e-mail: Jeffrey.Kieft@uchsc.edu; fax: (303) 724-3215.

Article and publication date are at http://www.rnajournal.org/cgi/doi/ 10.1261/rna.1360709.
Examples of tRNA mimicry are tRNA-like structures (TLSs) found at the $3^{\prime}$ end of certain positive-sense singlestranded RNA plant viruses (Fechter et al. 2001b; Dreher 2008). These viral RNAs are templates for protein synthesis, but they lack the poly (A) tail found on canonical mRNAs. In most cases, the TLS drives aminoacylation at the viral RNA's $3^{\prime}$ terminal CCA by a host cell aminoacyl tRNA-synthetase (AARS) (Fig. 1A; Pinck et al. 1970), and this activity led to their discovery and naming. In general, TLSs can be removed from their context in the viral genome and be aminoacylated (Joshi et al. 1982, 1983), suggesting that like many other functional RNAs, part of their function is contained in an independently formed structure. TLSs have been found that are valylated (Pinck et al. 1970), tyrosylated (Hall et al. 1972), or histidylated (Sela 1972); the significance of these amino acids versus others remains unknown. These three types of TLSs can also form functionally important interactions with other canonical tRNA-interacting proteins such as elongation factor $1 \mathrm{~A}$ (eEF1A). The efficiency of aminoacylation, the nature of protein binding, and the function of the TLS from different viruses can vary substantially. 
A

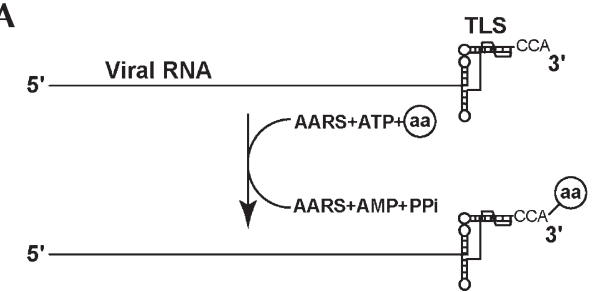

C

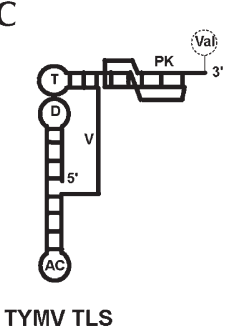

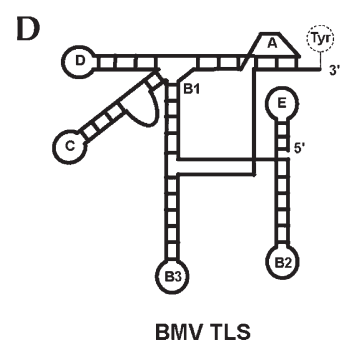

B

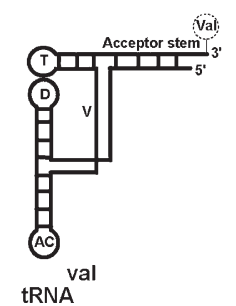

E

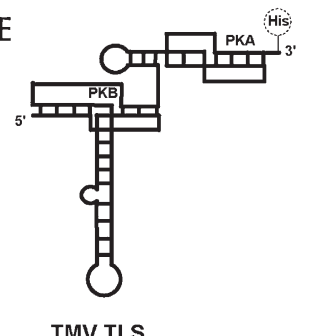

FIGURE 1. Secondary structures of tRNA and TLSs. (A) Schematic of a plant viral RNA with a TLS on the 3' end, which is aminoacylated (aa) by the host's enzyme (AARS). (B) Secondary structure cartoon for tRNA ${ }^{\text {Val }}$ with the T loop (T), D loop (D), anticodon loop (AC), variable arm (V), and acceptor stem labeled. (C) Secondary structure cartoon of the TYMV TLS. Structures analogous to those in tRNA are labeled, as is the pseudoknot (PK) in the acceptor stem. $(D)$ Cartoon of the secondary structure of the BMV TLS, with structural elements labeled. (E) Cartoon of the TMV TLS, with elements labeled. Consistent with previously published literature, all TLS elements are numbered beginning at the $3^{\prime}$ end.

The three TLS types are often represented by the valylated turnip yellow mosaic virus (TYMV) TLS, histidylated brome mosaic virus (BMV) TLS, and tyrosylated tobacco mosaic virus (TMV) TLS. The roles each play during infection vary, but in general, these include regulation/enhancement of translation, replication of the genome, fidelity of the $3^{\prime}$ end of the viral RNA, encapsidation, viral RNA genome stability, and thus overall virus amplification. Because they lie at the $3^{\prime}$ end of the viral genome, TLSs are among the first sequences to be replicated by the RNAdependent RNA polymerases (RDRPs) and can contain promoter sequences (Dreher et al. 1984; Singh and Dreher 1997; Osman et al. 2000); thus they could regulate the switch between negative strand synthesis and translation. In addition, one study proposes that the valylated TYMV TLS delivers its amino acid to the ribosome to initiate translation of a viral open reading frame in cis; this model remains controversial (Barends et al. 2003; Matsuda and Dreher 2007). The TLSs from different viruses perform diverse functions, thus all are multifunctional RNAs whose roles extend beyond recognition by an AARS, and it has been predicted that each possesses a specific three-dimensional (3D) structure that drives these multiple functions (Dumas et al. 1987; Felden et al. 1996, 1994) .

Despite some overlapping functions and the shared ability to be aminoacylated and to interact with eEF1A, the TYMV, BMV, and TMV TLSs differ dramatically in sequence, length, and predicted secondary structure. The minimal length of the TYMV TLS for aminoacylation has been reported as 82 nucleotides (nt) (Mans et al. 1990), the designated A, B1, B2, B3, C, D, and E (Fig. 1D), with some reports including an eighth $\mathrm{F}$ domain directly upstream of the TLS (Felden et al. 1994). The TMV TLS secondary structure is proposed to contain two pseudoknots (PK A and PK B). One of these pseudoknots may be analogous to the D-T loop interaction; however, no evidence of an actual D-T loop-loop interaction has been reported (Fig. 1E) (Rietveld et al. 1984). In addition, directly upstream of the TMV TLS is an upstream pseudoknot domain (UPD) (Zeenko et al. 2002). The ability of TLSs to mimic some functions of tRNAs has led to the idea that they possess a fold that mimics the classic tRNA structure, and chemical and enzymatic probing study results have been used to construct 3D structural models of the TYMV, BMV, and TMV TLSs that can be further evaluated and tested (Giege et al. 1990; Felden et al. 1994, 1996).

The diversity of sequences and proposed structures of these three representative TLSs raises the questions of how they are all recognized by AARSs and other tRNA-binding proteins, the degree to which their global architectures match tRNA and compare to each other, and how their structures contribute to their ability to perform multiple functions. To build on existing knowledge and address these questions, we explored the $3 \mathrm{D}$ architectures of the TYMV, BMV, and TMV TLS RNAs, using methods that explore the structure of each at multiple levels. Analytical ultracentrifugation, small-angle X-ray scattering (SAXS), and chemical probing illustrate clear differences in the global folding architectures of these TLSs when compared with each other and to tRNA, and show the specific 
interactions and structural dynamics at the heart of each fold that gives rise to this structural diversity. These results allow speculation as to how these TLSs act as multifunctional RNAs that regulate or drive various viral processes.

\section{RESULTS}

\section{Three representative TLSs adopt a compact fold}

To determine if three representative TLSs fold into homogeneous populations of compact molecules independently of the rest of the viral RNA, and to compare them with a tRNA, we analyzed in vitro transcribed TYMV (85 nt; TYMV-BL), BMV (169 nt; BMV-RNA3), TMV (106 nt; strain TMV-U5) TLSs, and Arabidopsis thaliana tRNA ${ }^{\mathrm{Val}}$, using sedimentation velocity analytical ultracentrifugation (SV/AUC). SV/AUC measures the rate at which molecules move through solution, reflecting hydrodynamic properties that depend on the molecule's size and shape. Thus, this method is useful for detecting changes in structure between a macromolecule's unfolded and folded states. Because RNA folds are stabilized by cations that neutralize the negatively charged backbone and allow close packing of the RNA, we conducted the SV/AUC both with and without $\mathrm{MgCl}_{2}$ to observe both the folded and unfolded states, respectively.

For all four RNAs, the resultant SV/AUC data were fit well by a single-species model, suggesting all four fold into structurally homogeneous populations. The fit data yield the model-independent measurements of the sedimentation (S) and diffusion (D) coefficients; both increase with $\mathrm{Mg}^{2+}$ for all the RNAs, indicating that addition of the divalent cation causes the molecules to become more compact (Table 1 ). The experimentally determined $S$ and $D$ values were used to calculate the Stokes radius $\left(R_{H}\right.$; radius of a sphere with the same molecular weight and same $S$ as the molecule of interest), and these values were compared with those from tRNA. For in vitro transcribed A. thaliana tRNA ${ }^{\mathrm{Val}}, \mathrm{R}_{\mathrm{H}}$ decreases by $41 \%$ upon folding. Similar changes were observed for the TMV TLS (39.2\%) and BMV TLS (38.4\%), although the TYMV TLS only showed a change of $26.5 \%$. This smaller change for TYMV likely reflects the smaller $\mathrm{R}_{\mathrm{H}}$ for the RNA in the absence of $\mathrm{Mg}^{2+}$; thus it is already somewhat compact under that condition. The calculated observed frictional coefficient to the frictional coefficient of a sphere $\left(\mathrm{f} / \mathrm{f}_{\mathrm{o}}=1\right.$ for a sphere $)$ reveals a substantial change between folded and unfolded states for all RNAs (A. thaliana tRNA ${ }^{\mathrm{Val}}$ : $1.90-1.21$, BMV: 2.50-1.49, TMV: 2.28-1.39; TYMV: 1.89-1.39) For all threefolded TLS species, the $\mathrm{f} / \mathrm{f}_{\mathrm{o}}$ is between 1.38 and 1.49 , showing that all three TLS RNAs respond to the presence of $\mathrm{Mg}^{2+}$ by undergoing a global structural compaction.

\section{SAXS reveals TLSs' overall molecular shapes}

Having determined that each representative TLS independently folds in the presence of $\mathrm{Mg}^{2+}$, we used SAXS to interrogate the structures in solution. All samples were passed through a gel filtration/size exclusion column monitored using dynamic light scattering (DLS) immediately before $\mathrm{X}$-ray exposure to ensure that the sample was monodisperse (Fig. 2A). The TYMV and BMV TLSs and tRNA ${ }^{\text {Phe }}$ were "well-behaved" samples that gave scattering curves amenable to further analysis, while the TMV TLS showed aggregation and structural heterogeneity under all conditions that we tested, precluding further biophysical analysis.

The SAXS profiles from yeast tRNA ${ }^{\text {Phe }}$, TYMV TLS, and BMV TLS were used to construct Kratky plots, which plot $\mathrm{I}(\mathrm{q})^{\star} \mathrm{q}^{2}$ versus $\mathrm{q}(\mathrm{q}$ reflects the scattering angle, and $\mathrm{I}(\mathrm{q})$ is the intensity of scattering at that angle). Protein and RNA molecules that are folded with internal structures produce a parabolic curve (indicating a self-association at smaller scattering distances), whereas molecules that are unfolded (and act more "rodlike") will have plots that continue to rise at higher $\mathrm{q}$ (smaller scattering distances). In the presence of $\mathrm{Mg}^{2+}$, all three exhibit an inverted parabola, showing they fold into a structured molecule with few or no unorganized regions (Fig. 2B). As expected, without $\mathrm{Mg}^{2+}$ they are less structured (data not shown). The tRNA and TYMV TLS Kratky plots are similar in shape with maxima in the same location, indicating similar global

TABLE 1. Analytical ultracentrifugation measurements

\begin{tabular}{|c|c|c|c|c|c|c|}
\hline \multirow[b]{2}{*}{ tRNA/TLS } & \multirow[b]{2}{*}{$\begin{array}{c}{\left[\mathrm{MgCl}_{2}\right]} \\
(\mathrm{mM})\end{array}$} & \multicolumn{2}{|c|}{ Measured values } & \multicolumn{3}{|c|}{ Calculated values } \\
\hline & & $\begin{array}{c}\mathrm{S}_{20, \mathrm{w}} \\
\text { (Svedbergs) }\end{array}$ & $\begin{array}{c}D_{20, w} \\
\left(\times 10^{7} \mathrm{~cm}^{2} / \mathrm{s}\right)\end{array}$ & $f / f_{o}$ & $\begin{array}{l}\mathrm{R}_{\mathrm{H}} \\
(\AA)\end{array}$ & $\begin{array}{c}\mathrm{R}_{\mathrm{H}} \\
\text { (\% change) }\end{array}$ \\
\hline tRNA ${ }^{\mathrm{Val}}$ & $\begin{array}{r}0 \\
10\end{array}$ & $\begin{array}{l}2.86 \pm 0.02 \\
4.50 \pm 0.03\end{array}$ & $\begin{array}{r}6.21 \pm 0.06 \\
12.25 \pm 0.21\end{array}$ & $\begin{array}{l}1.90 \\
1.21\end{array}$ & $\begin{array}{l}32.2 \\
19.0\end{array}$ & $41.0 \%$ \\
\hline TYMV TLS & $\begin{array}{r}0 \\
10\end{array}$ & $\begin{array}{l}3.09 \pm 0.03 \\
4.21 \pm 0.06\end{array}$ & $\begin{array}{l}6.14 \pm 0.24 \\
8.66 \pm 0.61\end{array}$ & $\begin{array}{l}1.90 \\
1.40\end{array}$ & $\begin{array}{l}31.0 \\
22.7\end{array}$ & $26.6 \%$ \\
\hline TMV TLS & $\begin{array}{r}0 \\
10\end{array}$ & $\begin{array}{l}2.99 \pm 0.0 \\
4.92 \pm 0.06\end{array}$ & $\begin{array}{l}5.25 \pm 0.26 \\
9.54 \pm 0.10\end{array}$ & $\begin{array}{l}2.28 \\
1.39\end{array}$ & $\begin{array}{l}43.3 \\
26.3\end{array}$ & $39.2 \%$ \\
\hline BMV TLS & $\begin{array}{r}0 \\
10\end{array}$ & $\begin{array}{l}3.71 \pm 0.06 \\
6.23 \pm 0.03\end{array}$ & $\begin{array}{l}3.75 \pm 0.14 \\
6.52 \pm 0.38\end{array}$ & $\begin{array}{l}2.50 \\
1.49\end{array}$ & $\begin{array}{l}51.5 \\
31.7\end{array}$ & $38.4 \%$ \\
\hline
\end{tabular}


A

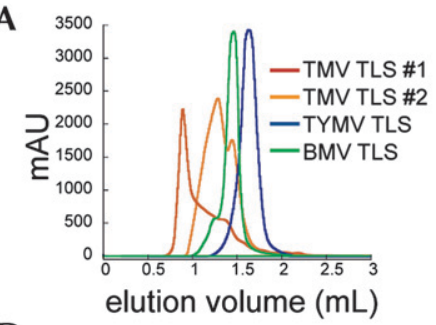

D

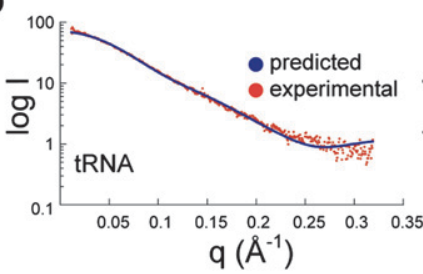

B

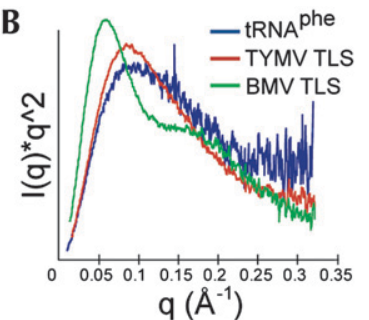

C

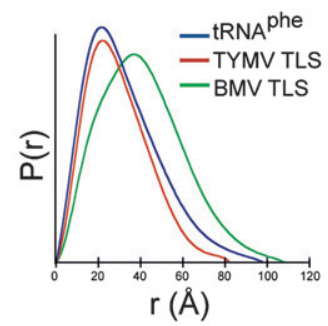

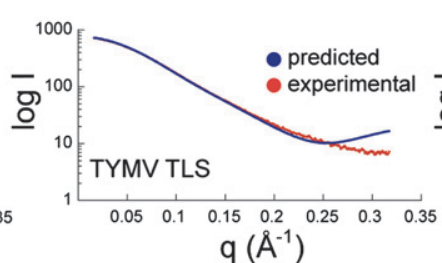

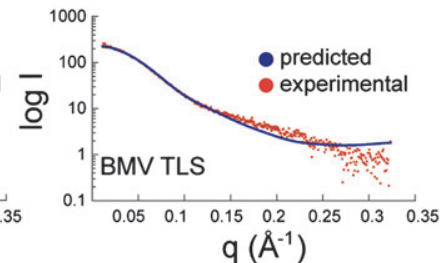

$\mathbf{E}$
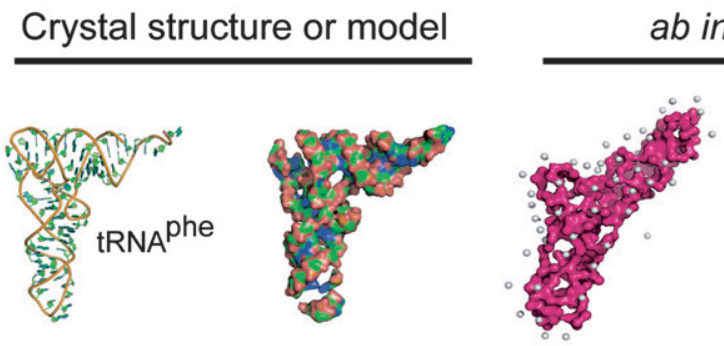

ab initio SAXS reconstructions
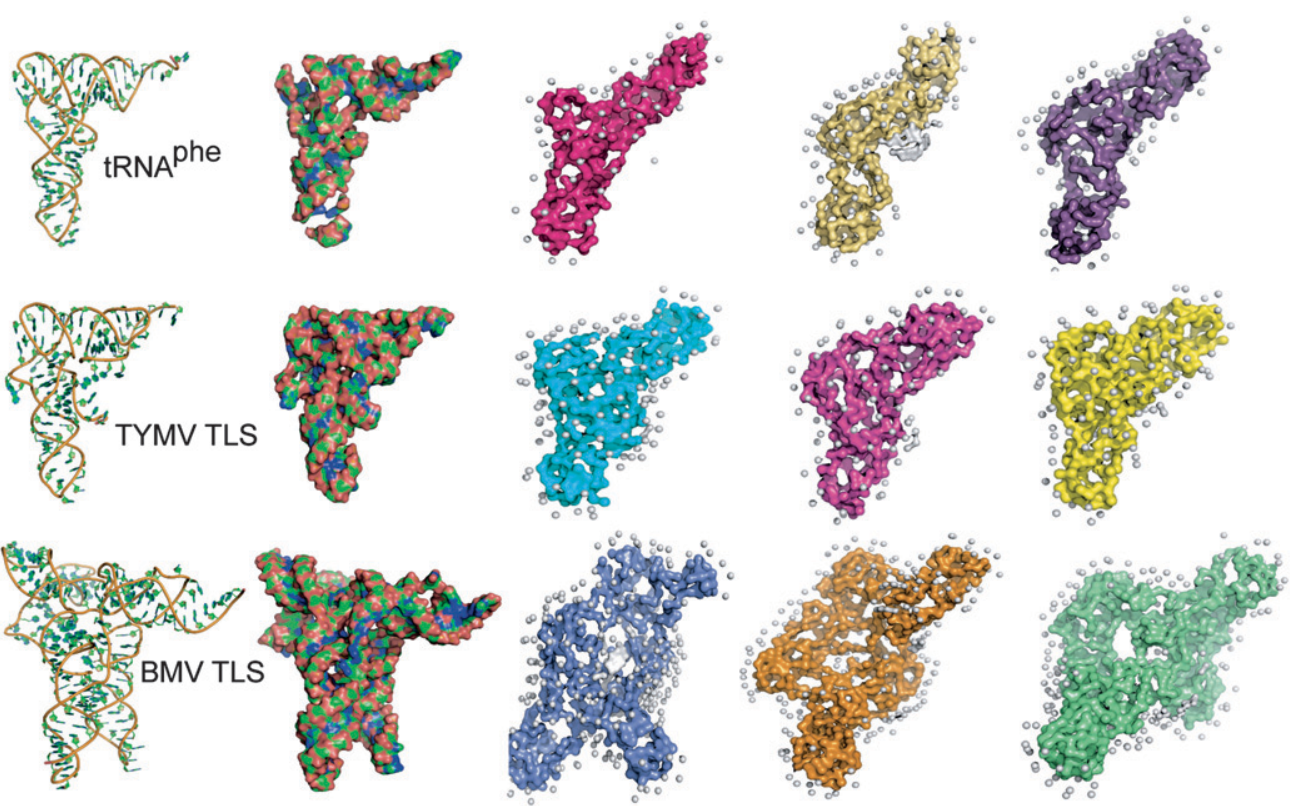

FIGURE 2. SAXS of tRNA and TLSs. (A) Gel filtration analysis of the TYMV (blue), BMV (green), and TMV TLS (red and orange), with the elution monitored by dynamic light scattering (DLS). All traces are of RNA in the presence of $\mathrm{Mg}^{2+}$. The TYMV and BMV TLSs elute as a single major peak consistent with their molecular weight and size. Traces of TMV TLS RNA annealed by two different procedures both show broad elution profiles at locations consistent with multimerization and heterogeneity. (B) Kratky plots of the scattering data of yeast tRNA ${ }^{\text {Phe }}$ (blue), the TYMV TLS (red), and the BMV TLS (green). (C) Graph of the pairwise distribution function for each RNA; colors match panel B. $(D)$ Comparison of the experimental scattering profile (red) of tRNA ${ }^{\text {Phe }}$, the TYMV TLS, and the BMV TLS compared with the predicted scattering profile (blue) calculated from the crystal structure of tRNA ${ }^{\text {Phe }}$ (Protein Data Bank 1EHZ), and the structural models of the two TLSs. (E) At left are the crystal structure of yeast tRNA ${ }^{\text {Phe }}$ and models of the structures of the TYMV and BMV TLSs. To the right of this is a space-filling model of each structure/model, and further to the right are three representative independently calculated ab initio reconstructions using the GASBOR modeling program (Svergun et al. 2001). These representative reconstructions were chosen purposely to represent the full variability observed. White spheres represent modeled waters in the hydration sphere.

molecular conformations. The measured radius of gyration $\left(\mathrm{R}_{\mathrm{G}}\right)$ for yeast tRNA ${ }^{\text {Phe }}(25.6 \AA)$ is slightly larger than that of the TYMV TLS (23.1 $\AA$ ), despite the fact that the tRNA has a smaller molecular mass; this might indicate tighter packing in the TYMV TLS (Table 2). In contrast, the plot of the BMV TLS Kratky plot exhibits a different shape and the BMV TLS has a larger $\mathrm{R}_{\mathrm{G}}(31.9 \AA)$, showing that the BMV
TLS conformation in solution is substantially different from these other RNAs, consistent with published models.

The SAXS profiles of the tRNA, TYMV TLS, and BMV TLS were used to generate a plot of the pairwise distribution function, showing the distribution of distances between scattering electron pairs in the molecule and the maximum physical dimension $\left(\mathrm{D}_{\max }\right)$ of the molecule at the nonzero 
TABLE 2. SAXS measurements and parameters used

\begin{tabular}{|c|c|c|c|}
\hline tRNA/TLS & $\begin{array}{l}\mathrm{R}_{\mathrm{G}} \\
(\AA)\end{array}$ & $\begin{array}{c}\mathrm{D}_{\max } \\
(\AA)\end{array}$ & Dummy residues used \\
\hline tRNA ${ }^{\text {Phe }}$ & 25.6 & 98 & 269 \\
\hline TYMV TLS & 23.1 & 82 & 298 \\
\hline BMV TLS & 31.9 & 108 & 473 \\
\hline
\end{tabular}

$\mathrm{X}$ intercept (Fig. 2C). For yeast tRNA ${ }^{\text {Phe }}$ and the two TLSs, these plots indicate a large portion of the electron scattering pairs are located $\sim 20 \AA$ apart, coinciding with the width of A-form RNA helices. The curves of yeast tRNA ${ }^{\text {Phe }}$ and the TYMV TLS are similar, but differ in their $\mathrm{D}_{\max }$ (Table 2). Specifically, the maximum dimension of tRNA ${ }^{\text {Phe }}$ is $98 \AA$, larger than the TYMV TLS ( $82 \AA)$. In contrast to tRNA and the TYMV TLS, the curve from the BMV TLS has a maximum at $40 \AA$ and a shoulder at $\sim 20 \AA$. This suggests not only the formation of A-form helices, but also that more scattering pairs are farther apart than in tRNA, which is consistent with a collection of packed helices. The BMV TLSs maximum dimension of $108 \AA$ is only $10 \AA$ larger than that measured for tRNA, although the molecule contains more than twice the number of nucleotides (169 versus $\sim 75$ ). This indicates dense RNA backbone packing within the BMV structure to a degree that is greater than that found in classic L-shaped tRNA.

\section{Testing published models with SAXS}

Each molecular structure yields a distinct SAXS profile; thus, we can test published TLS and BMV TLS structural models by comparing the scattering predicted from the model with the experimental scattering (Fig. 2D). Using tRNA as a control, the curve predicted from the crystal structure (using the program CRYSOL) matches the experimentally measured scattering profile with some deviation at the highest scattering angles $\left(\mathrm{q}>0.25 \AA^{-1}\right)$ where signal to noise is low and a slight deviation at the lowest scattering angles (extreme left of curve), perhaps due to some residual aggregates. This shows that the crystal structure of tRNA is a good representation of the global conformation of the molecule in solution. For the TYMV TLS model, the fit between the predicted and experimental scattering is good, although there is more deviation in the higher scattering angles. BMV TLS scattering deviates noticeably from that predicted from the model at $\mathrm{q}>$ $0.15 \AA^{-1}$. These data indicate that the model of the TYMV TLS is reasonable but not completely correct, but the BMV TLS model is substantially different from the true global solution conformation.

\section{D SAXS reconstructions of TLSs}

The X-ray scattering curves of the TLSs and tRNA provide a direct experimental way to compare these molecules to each other. However, using these curves to generate models that are consistent with the scattering provides a way to visually assess how different their physical shapes may be from one another. Therefore, we used the program GASBOR to calculate ab initio reconstructions of each molecule using the SAXS data independent of prior assumptions about their shape (Svergun et al. 2001). To identify features conserved among the reconstructions, multiple reconstructions were generated independently for each RNA; Figure $2 \mathrm{E}$ represents the full range of variability in the reconstructions. As a control, we again used tRNA. Multiple reconstructions of both yeast tRNA ${ }^{\text {Phe }}$ (Fig. 2E) and $A$. thaliana tRNA ${ }^{\text {Val }}$ (data not shown) produced shapes reminiscent of two helices meeting at an angle, but with a more elongated conformation than that found in a highresolution tRNA ${ }^{\text {Phe }}$ crystal structure (Friederich et al. 1998; Shi and Moore 2000). However, application of various methods shows variability in the measured helical angles in the tRNA elbow (Friederich et al. 1998). For the purposes of this study, we did not concentrate on the precise angle between these helices, but rather on how well the predicted global shape of the TLS RNAs compared with the predicted global shape of the tRNA. TYMV TLS reconstructions were consistent in that all comprise a shape similar to tRNA's, but the reconstructions of the BMV TLS do not match the shape of tRNA or the TYMV TLS and are more variable from each other (Fig. 2E). Despite variability in the details, the BMV TLS reconstructions all show a central globular region with helix-sized protrusions, consistent with a core of packed helices, with loops E, B2, C, D, or the acceptor stem extending outward. This type of architecture is similar in type to that predicted by the published model (Fig. 2E), although none of the reconstructions match this model well.

\section{TYMV TLS: Tight backbone packing creates the global architecture}

Our solution biophysical analyses reveal global characteristics of the TYMV and BMV TLSs; the next step was to investigate the local backbone packing interactions that are the determinants of these three-dimensional structures, beginning with the TYMV TLS. Hydroxyl radical $(\bullet \mathrm{OH})$ probing is different from most other chemical and enzymatic probing methods. $\bullet \mathrm{OH}$ probing interrogates the solvent accessibility of the RNA backbone at each location, and the pattern of protection reveals the degree to which different parts of the molecule are packed together within the fold. Previously published hydroxyl radical probing of yeast $\mathrm{tRNA}^{\text {Phe }}$ shows protections in the $\mathrm{D}, \mathrm{T}$, and variable loops, and this pattern is a "fingerprint" of the classic tRNA fold (Fig. 3A; Latham and Cech 1989). To verify this "fingerprint," we probed in vitro transcribed A. thaliana tRNA Val, observing expected protections in the $\mathrm{D}, \mathrm{T}$, and variable loops and also previously 
A

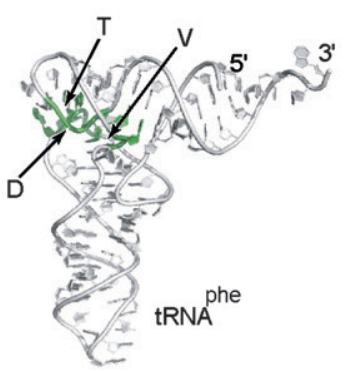

$\mathbf{E}$

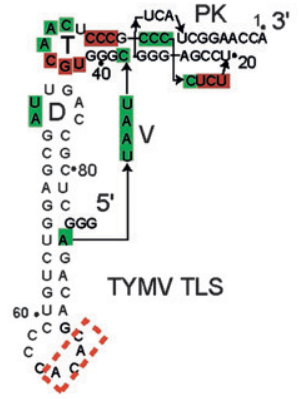

B

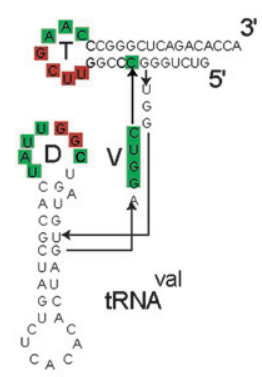

$\mathbf{F}$

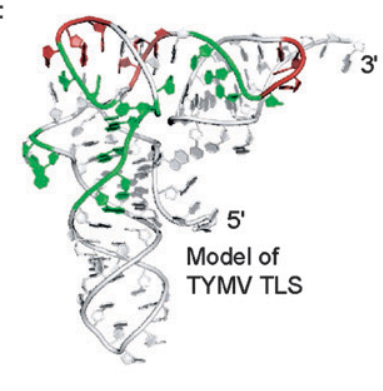

C

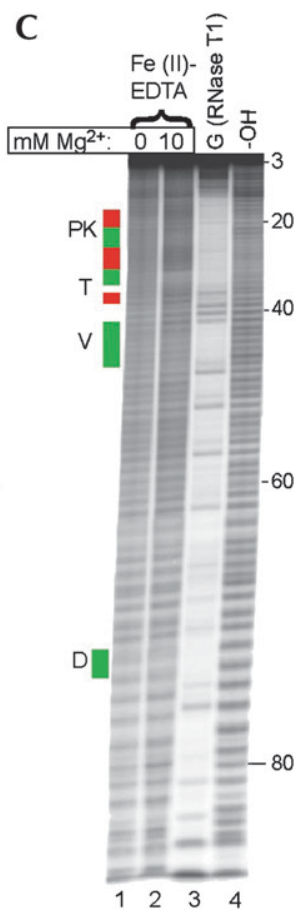

D

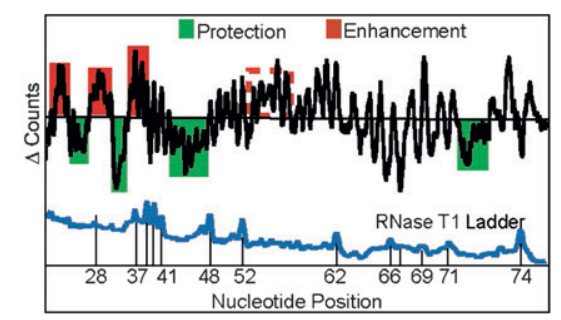

FIGURE 3. Hydroxyl radical probing of tRNA and TYMV TLS. (A) Crystal structure of yeast tRNA ${ }^{\text {Phe }}$ (Protein Data Bank 1EHZ) with hydroxyl radical protections (green) observed by Latham and Cech (1989) overlaid. (B) Hydroxyl radical protection (green) and enhancement (red) observed for A. thaliana tRNA ${ }^{\mathrm{Val}}$; raw gel is not shown. (C) Representative hydroxyl radical probing experiment of TYMV TLS, lanes 1,2 are probed without and with $\mathrm{Mg}^{2+}$, respectively. Secondary structure features are indicated left of gel, and nucleotide position on right. Green bars indicate areas of protection, and red indicates areas of enhanced cleavage with $\mathrm{Mg}^{2+}$. Lanes 3,4 contain an RNase T1 ladder (G) and a hydrolysis ladder, respectively. (D) Quantification of part of the gel in panel C. Graph of the difference of counts found in lane 2 subtracted from lane 1 as a function of nucleotide position on the bottom. Areas above the "zero" line represent areas of backbone cleavage enhancement (red), while areas below the line represent areas of protection (green). Blue line is T1 ladder lane trace with corresponding nucleotide position. (E) Secondary structure diagram of TYMV TLS with protections and enhancements overlaid in green and red, respectively. Dotted red line indicates area of subtle yet reproducible cleavage enhancement. (F) Observed hydroxyl radical protections (green) and enhancements (red) mapped onto a published model of the TYMV TLS.

unreported cleavage enhancements in the $\mathrm{D}$ and $\mathrm{T}$ loops (Fig. 3B).

Upon addition of $\mathrm{Mg}^{2+}, \bullet \mathrm{OH}$ probing of the TYMV TLS shows a cleavage pattern similar to tRNA's, but with several differences (Fig. 3C,D). Specifically, solvent protection is found in the $\mathrm{D}, \mathrm{T}$, and variable loops, and a subtle cleavage enhancement is found in the anticodon loop (Fig. 3E), strongly suggesting that some interactions within these parts of the fold mimic tRNA. However, the amount of protection and enhancement in the D loop of the TYMV TLS is less than in A. thaliana $\mathrm{tRNA}^{\mathrm{Val}}$, suggesting this loop packs less tightly into the "elbow" of the L-shaped structure. Also, the TYMV TLS acceptor stem pseudoknot shows both cleavage protections and enhancements, consistent with the complex fold revealed in its NMR structure (Kolk et al. 1998). Mapping hydroxyl radical probing data onto the model of the TYMV TLS RNA (for review, see Fechter et al. 2001b) shows many protections cluster together near the putative elbow region, but many of the regions we find to be protected are solvent exposed in the model, suggesting local structural differences between the TYMV TLS and tRNA (Fig. 3F).

\section{TYMV TLS: Determinants of the fold include 5 ' nucleotides}

To further understand the local determinants of the TYMV TLSs global fold, we explored the role of $5^{\prime}$ sequences. The TYMV TLS sequences used in the above probing study contains $85 \mathrm{nt}$ (initially, we used a $5^{\prime}$ GGG sequence to promote efficient in vitro transcription followed by 82 viral nucleotides and no upstream ribozyme), as previous aminoacylation assays identified $82 \mathrm{nt}$ as comprising the functional TLS. However, there is some ambiguity regarding this boundary and the effects of $5^{\prime}$ sequences on the TLS structure (Joshi et al. 1982; Dreher et al. 1988; Mans et al. 1990). To systematically explore the structural role of the $5^{\prime}$ end, we $\bullet \mathrm{OH}$ probed four RNAs with systematic variation of the number of G's on this terminus. The first RNA, containing the last $82 \mathrm{nt}$ of the viral genome (no added G's), shows no cleavage enhancements or protections with $\mathrm{Mg}^{2+}$ (Fig. 4A), and hence, no evidence of a tightly packed structure. Addition of one $5^{\prime} \mathrm{G}(83 \mathrm{nt})$ results in subtle protection in the $\mathrm{T}$ loop and variable arm (Fig. 4A,B), suggesting this base specifically stabilizes an 
A

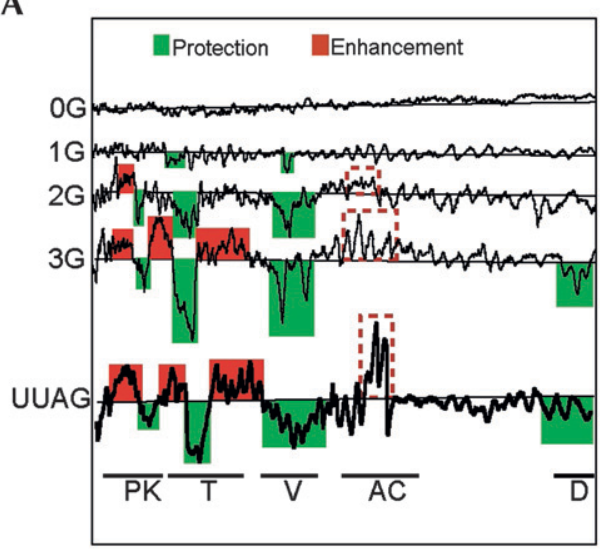

C

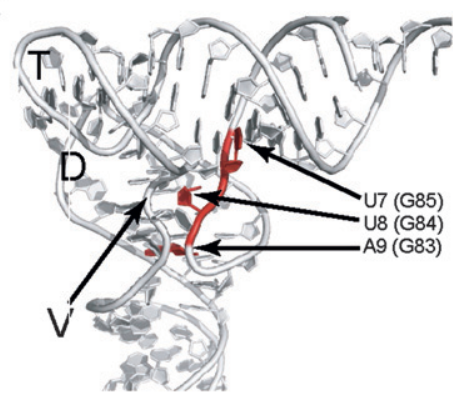

B

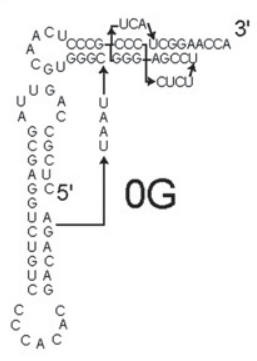

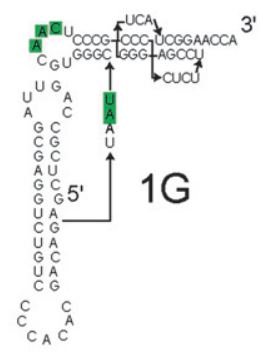

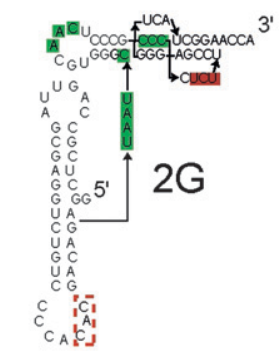

FIGURE 4. Influence of $5^{\prime}$ nt on the structure of TYMV TLS. (A) Quantified hydroxyl radical probing results of five variants of TYMV TLS. The identity of each RNA is shown on the left, and diagrams of variants $0 \mathrm{G}, 1 \mathrm{G}, 2 \mathrm{G}$, and $3 \mathrm{G}$ are shown in panel $B$, this figure. The "UUAG" variant contains the native viral RNA sequences at the 5' end. As in Figure 2, locations where the trace is above the zero line correspond to locations in the backbone with enhanced cleavage (red), those below are protected from cleavage (green), and the red dotted line indicates subtle but reproducible cleavage enhancement. Secondary structure regions are indicated at the bottom. (B) Secondary structure diagrams of the four TYMV TLSs with different $5^{\prime}$ ends. Hydroxyl radical protections and enhancements are indicated on each with green and red, matching panel A. $(C)$ Close-up view of part of the structure of yeast tRNA ${ }^{\text {Phe }}$, with nucleotides labeled and U7-A9 highlighted in red. The locations of the T loop, D loop, and variable loop are shown.

interaction between those two elements, but that the $\mathrm{D}$ loop and pseudoknot remain unpacked. Addition of two G's (84 nt) results in additional protections and enhancements in the acceptor stem pseudoknot, suggesting that the pseudoknot, $\mathrm{T}$ loop, and variable arm are organized without the D loop. Addition of the third G (85 nt) results in the full set of protections and enhancements, including the D loop. Having noted the effects of adding G-bases successive to the $5^{\prime}$ end, we then probed a TYMV TLS RNA that containing the native $5^{\prime}$-UUAG sequence (generated by removing the 5' GGG sequence and replacing it with an upstream self-cleaving ribozyme). The pattern of protection matched that observed with 3G's (Fig. 4A, bottom trace). The progressive protection of the TYMV as the $5^{\prime}$ end is lengthened shows that nucleotides $5^{\prime}$ of the "minimal" TYMV TLS perform critical roles in stabilizing the tightly packed backbone within the tertiary structure.

A hypothesis for how three $5^{\prime}$ nucleotides might stabilize the overall tertiary structure of the TYMV TLS is suggested

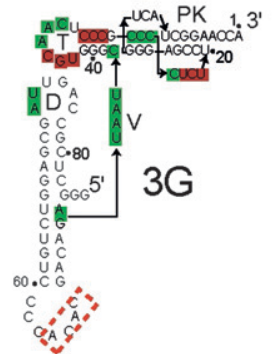

by examination of the crystal structure of a tRNA, where nucleotides between the anticodon and acceptor stems interact with bases in the D loop, T loop, and acceptor stem (Shi and Moore 2000). Specifically, in yeast tRNA ${ }^{\text {Phe }}$, U9 forms a base triple with A23 and U12 (D stem) and stacks between G45 and 7mG46 (variable loop), and U8 interacts with A14 (D loop), and U7 pairs with A66 (acceptor stem) and stacks on 5mC49 (T stem) (Fig. 4C). We hypothesize that TYMV TLS nucleotides 83-85 analogously act to stabilize these central interactions in the core of the fold. Dreher et al. (1988) previously speculated that nucleotides 83-84 might interact with U12 of the TYMV TLS acceptor stem pseudoknot, and a model in which the $5^{\prime}$ end is positioned to make these interactions was proposed by Rietveld et al. (1983), but models in which the $5^{\prime}$ end was extending away from the molecule have also been depicted (Fig. 3F; Dumas et al. 1987). Our data suggest a possible role for these nucleotides, acting as a "zipper" and stabilizing the overall fold, a model that can be tested in future studies.

\section{TYMV TLS: Dynamic regions include the acceptor-stem pseudoknot}

Hydroxyl radical probing shows locations of the RNA backbone that are protected from solvent, and hence, tightly packed, but does not reveal patterns of base-pairing or local dynamic behavior. Selective 2'-hydroxyl acylation analyzed by primer extension (SHAPE) is a probing method that assays secondary structure and local dynamics (Merino et al. 2005) and thus provides structural information complimentary to $\bullet \mathrm{OH}$ probing. We therefore used SHAPE to explore additional details of the folded TYMV TLS. As a control, we probed A. thaliana tRNA ${ }^{\mathrm{Val}}$ and obtained a pattern that agreed with published SHAPE studies on yeast tRNA ${ }^{\text {Asp }}$ (Fig. 5A; Merino et al. 2005). SHAPE of the TYMV TLS is similar to tRNA, but many additional modifications are in the acceptor stem pseudoknot (Fig. 5B,C). This pseudoknot is reported as relatively unstable, and NMR studies required several mutations (near and in the T loop) to stabilize the structure enough to allow structure determination (Kolk et al. 1998). Thus, this pseudoknot is likely stabilized by the rest of 
A

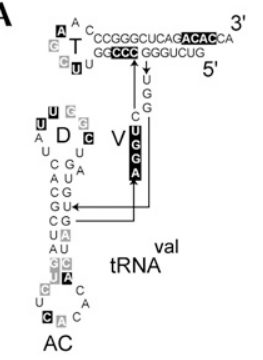

C

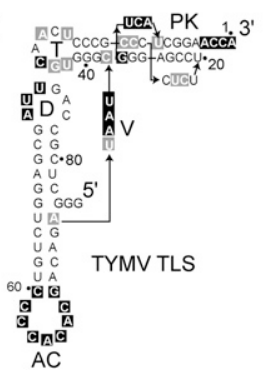

B

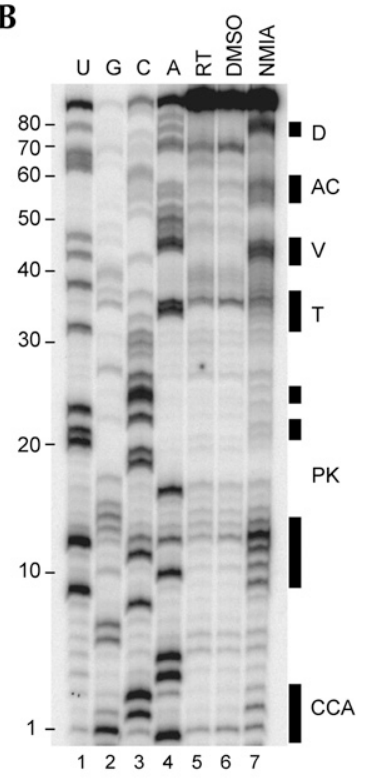

FIGURE 5. Selective $2^{\prime}$ hydroxyl acylation analyzed by primer extension (SHAPE) of tRNA and TYMV TLS. (A) SHAPE results of A. thaliana tRNA $^{\mathrm{Val}}$ mapped onto its secondary structure diagram. Modified bases are boxed gray (weak) or black (strong). (B) Representative SHAPE experiment of TYMV TLS. Lanes 6,7 are without and with acylating agent N-Methylisatoic Anhydride (NMIA), respectively. Lanes 1-4 are ddDNTP sequencing ladders. Nucleotide positions are to the left, and the locations of secondary structure features are indicated on the right. (C) SHAPE experiment results mapped onto secondary structure diagram of TYMV TLS. Shading matches panel $A$.

the folded TLS but remains flexible ("breathes") even in the context of the entire molecule.

\section{BMV TLS: The structure is created around a tightly folded multihelix core}

As with the TYMV TLS, we investigated the determinants of the global architecture of the BMV TLS fold that was revealed by SV/AUC and SAXS. $\bullet$ OH-probing of BMV TLS domains A-E (nucleotides 1-169) in the absence and presence of $\mathrm{Mg}^{2+}$ show that $\mathrm{Mg}^{2+}$-dependent protections and enhancements include $\sim 50 \%$ of the molecule, indicating formation of a tightly packed structure involving much of the RNA backbone (Fig. 6A,C). Specifically, protections are found in and around the four-way junction, to include B1, the base of $\mathrm{C}$, part of a bulge in $\mathrm{C}$, and the B2-3' end connection. These protections suggest these regions are a core around which the rest of the molecule packs, but they do not follow the standard D-loop/T-loop pattern seen in tRNA and the TYMV TLS, suggesting a very different core architecture. In addition, cleavage enhancements in the apical D, C, and B2 loops show they extend into solution, and some small enhancements adjacent to protected areas near the junction suggest complex packing

in this core. Protections and enhancements also are found in the acceptor stem pseudoknot at the $3^{\prime}$ end of the BMV TLS. The overall $\bullet \mathrm{OH}$ probing pattern of the BMV TLS is consistent with models of the BMV TLS that include a packed core, but parts of the molecule that are solvent protected in our probing experiments are exposed in the model (Fig. 6E). For example, our probing shows subtle but reproducible protection in loop B3 (Fig. 6A), as well as a tentative enhancement in this same loop. A strong site of "background" hydrolysis occurs in this loops that makes analysis difficult, but this cleavage pattern suggests that this loop packs in or against the core fold (perhaps transiently) and that other parts of the molecule not previously known to pack against the core may be part of an intricate central fold.

\section{BMV TLS: A negative strand promoter sequence shows dynamic behavior}

SHAPE analysis of the BMV TLS showed many of the predicted single-stranded regions are modified, including nucleotides located in the apical loops of elements D, C, B2, and the single-stranded region between B2 and A domains (Fig. 6B,D). There is less modification in the acceptor stem pseudoknot of the BMV TLS than in the pseudoknot of the TYMV TLS, indicating it is a more stable structure in this TLS than in TYMV. In addition, a bulge in the $\mathrm{C}$ stem that is part of the promoter for (-) strand synthesis by the RDRP is modified and hence is has some dynamic character. The $\bullet \mathrm{OH}$ probing shows this bulge has parts with enhanced cleavage and protection, indicating it lies on the interface between the folded core and the solvent exposed exterior of the molecule (Fig. 3C). Previous studies show that stabilizing mutations to this region inhibit (-) strand synthesis, hence this dynamic behavior within the folded RNA may have functional significance (see Discussion).

\section{TMV TLS: No evidence of tight backbone packing}

Structural models of the TMV TLS have PK B mimicking the D-T loop interaction of classic tRNA, allowing an Lshaped tRNA-like structure (Felden et al. 1996). We conducted $\bullet \mathrm{OH}$ probing of this RNA, but this experiment revealed no detectable backbone protections or enhancements, even with elevated $\mathrm{Mg}^{2+}$ concentrations of $20 \mathrm{mM}$ (data not shown). This result appears at odds with our SV/ AUC data showing that the TMV TLS compacts upon addition of $\mathrm{Mg}^{2+}$ (Table 1). These two results can be reconciled with the interpretation that while addition of $\mathrm{Mg}^{2+}$ leads to a global change in shape upon formation of secondary and some tertiary structure (as monitored by SV/AUC), there is no local packing of RNA backbone sufficiently tight to exclude solvent (as monitored by $\bullet \mathrm{OH}$ probing). There is precedent for this in other RNAs where 
A

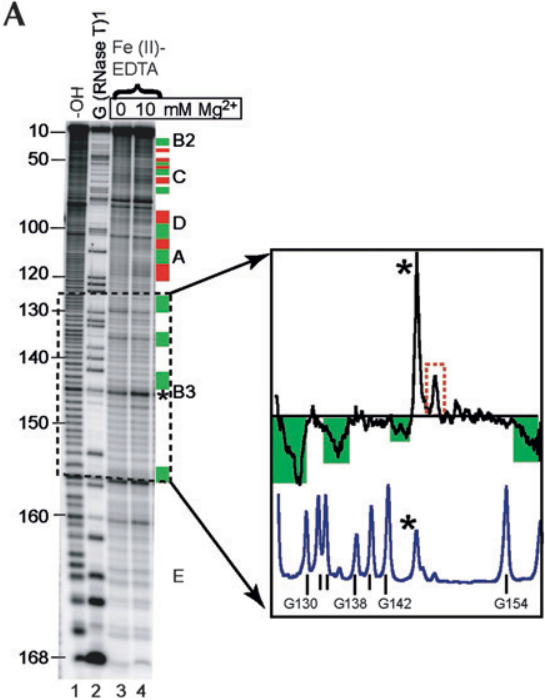

$\mathbf{E}$
B

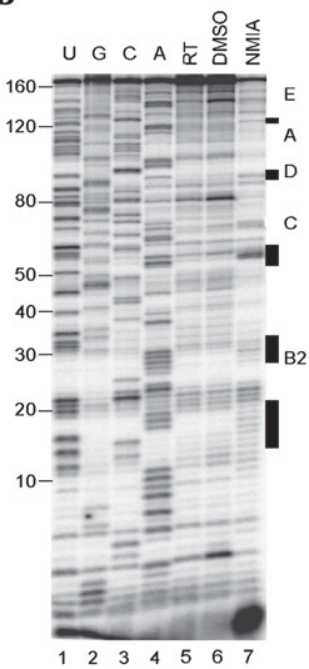

C
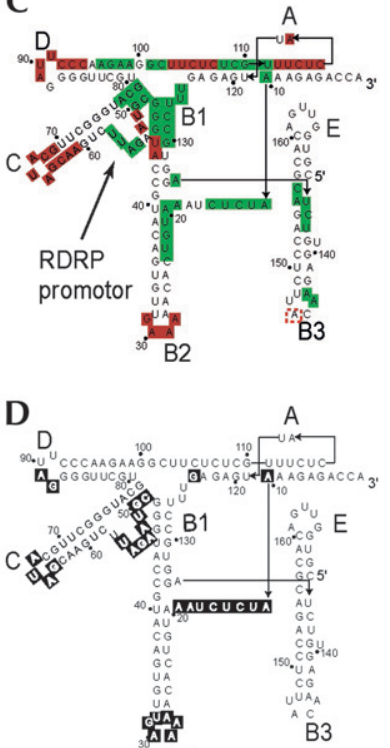

B2

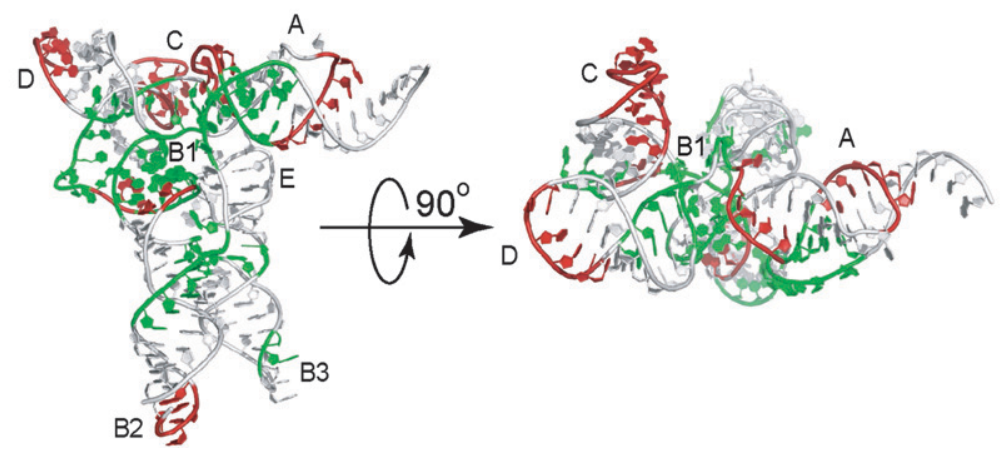

FIGURE 6. Probing of the BMV TLS. (A) Representative hydroxyl radical probing experiment of the BMV TLS. Hydrolysis ladder is in lane 1; RNase T1 probe, in lane 2; and hydroxyl radical cleavage without and with $\mathrm{Mg}^{2+}$, in lanes 3,4 , respectively. Nucleotide positions are indicated on the left; areas of protection (green) and enhancement (red), on the right; and secondary structure elements, to the far right. To the right of the gel is a quantitated and normalized trace of the boxed portion of the gel, showing the subtle but reproducible protection within loop B3. The asterisks denote a location in the backbone that is very prone to hydrolysis, and hence, appears as a peak in all lanes, preventing analysis of this particular position. (B) Representative gel of a SHAPE experiment for the BMV TLS. Lanes 1-4 are sequencing ladders, lane 5 is an RT control lane, and lanes 6 and 7 are without and with NMIA, respectively. Nucleotide positions are shown on the left, and secondary structure elements and areas of modification are indicated on the right. (C) Secondary structure of BMV TLS with hydroxyl radical protections (green) and enhanced cleavages (red) observed in panel $A$ mapped on to the diagram. The dashed red box indicates a tentative enhancement. $(D)$ Secondary structure diagram of BMV TLS with weak modifications in gray and strong modifications in black. Note that some modifications are not readily visible by eye in panel $B$ but were assigned through multiple repetitions of this experiment with quantification and normalization. (E) Hydroxyl radical probing results displayed on a published structural model of the BMV TLS with a front view, and rotated $90^{\circ}$ for top view. Secondary structure elements are labeled and colors match panels $A$ and $C$.

initial compaction the RNA as monitored by SV/AUC is separate from tight backbone packing as observed by $\bullet \mathrm{OH}$ probing (Costantino and Kieft 2005). SHAPE of the TMV TLS shows modifications in regions predicted to be single stranded but also in PK B1 (Fig. 7A,B). Thus, under these conditions, we find no evidence for a stable compact fold in the unbound, isolated TMV TLS. It is possible that additional RNA sequence, the AARS, or altered buffer/salt conditions are necessary for stabilization. Previous studies have noted that TMV TLS from different strains are aminoacylated with different efficiencies (Garcia-Arenal 1988), as if some get trapped in an inactive or misfolded state.

\section{DISCUSSION}

Positive-sense RNA viruses have subtle, complex, and elegant ways to co-opt the host cell's machinery, coordinate various viral processes, evade antiviral responses, and ultimately produce more viral particles. Many of these tasks depend on specifically folded nonprotein coding RNA structures within the viral RNA genome, and one example of this is the family of plant viral TLSs. The ability of these TLSs to be aminoacylated and to interact with other tRNA binding proteins led to the prediction that despite their different sequences and secondary structures, certain 3D 
A
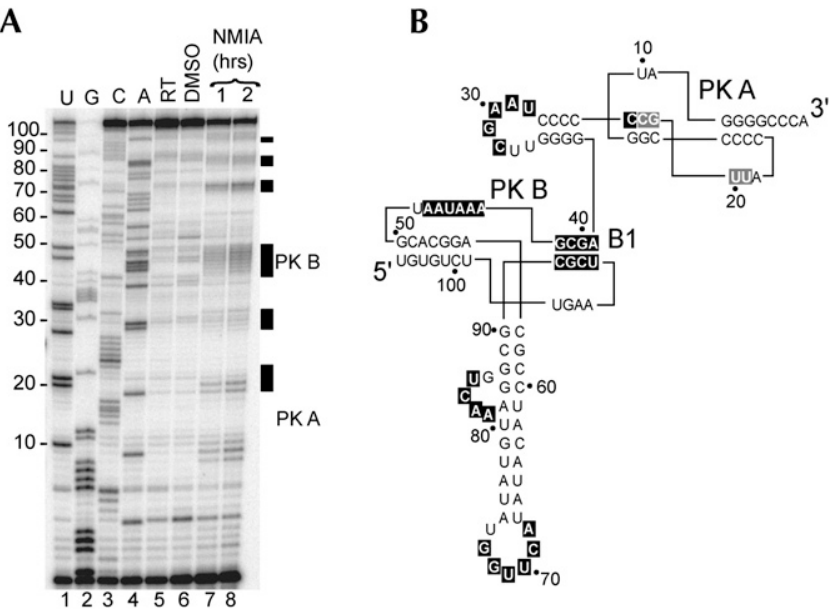

FIGURE 7. SHAPE of the TMV TLS. (A) Representative SHAPE experiment of the TMV TLS. Lanes $1-4$ are sequencing ladders, lane 5 is an RT control lane, lane 6 is a no-NMIA control lane, and lanes 7,8 contain NMIA with 1- and 2-h incubations, respectively. Two time points were used to detect effects of a longer incubation time. Locations of PK A and PK B are shown on the right; nucleotide positions, on the left. (B) Mapping of SHAPE data onto a TMV TLS secondary structure. Weak modifications are in gray and strong modifications in black. Note that some modifications are not readily visible by eye in panel $A$ but were assigned through multiple repetitions of this experiment with quantification and normalization.

structural characteristics would be found in each, such as the classic L-shaped tRNA structure. In this study, our strategy was to use a combination of methods to first study the global conformations of representative TLSs and then to explore the detailed interactions that give rise to the structures, making no assumptions about the structure of these molecules at any level. As such, these studies expand on previous studies in which enzymatic and chemical probing of TLS secondary and tertiary structure interactions and the ability of these RNAs to functionally mimic tRNAs were used to generate 3D models (Rietveld et al. 1983, 1984; Dumas et al. 1987; Felden et al. 1994, 1996; Fechter et al. 2001a) and also provide an independent testing of these models. Our results show that, consistent with previous studies, there are features of these TLSs that are reminiscent of tRNAs but also substantial diversity in their folds when compared with each other and with tRNA, and also reveal details of the folds and how they may act as multifunctional RNAs important for several tasks during viral infection.

In these studies, we employed SAXS, which is a biophysical technique that is relatively rarely used to study RNA global structure. SAXS cannot yield detailed structural information in the same way as high-resolution methods, but the scattering curves for a given macromolecule is very sensitive to its global characteristics and hence provides an independent way to interrogate the structure with no prior assumptions. Differences in experimentally measured scattering curve indicate true differences in size and shape, and we used this to compare the TLSs and tRNA with each other and with published structural models. The TYMV TLS has scattering characteristics similar to a tRNA and previous models, while the BMV TLS scattering profile is markedly different from a tRNA and also shows deviation from a previous model, suggesting this model can be refined. SAXS data can also be used to generate $3 \mathrm{D}$ reconstructions of molecular shapes consistent with the experimental scattering curves. These SAXS-derived reconstructions are most useful when combined with other methods rather than as a stand-alone method to unambiguously determine detailed molecule shapes. Hence, in this study we used reconstructions to assess the range and type of RNA conformations that could give rise to the experimental scattering curves obtained from TLS molecules. For the TYMV TLS, multiple independent reconstruction calculations produced a set of shapes with similar characteristics, suggesting that (1) there is one shape consistent with the scattering data; (2) this shape is tRNA-like; and (3) previous models for the TYMV TLS structure largely are correct. In the case of the BMV TLS, we obtained a greater variation between independently calculated reconstructions, all of which could produce the experimental scattering curve. Hence, while we cannot make unambiguous conclusions regarding the details of the shape of the BMV TLS, the reconstructions are consistent with a structure comprising a central globular domain with protrusions, consistent with probing data (Felden et al. 1994). Taken together, our SAXS data show that this method is useful to assess and compare RNA structures for which no high-resolution structural information is available, which can in turn help develop new hypotheses, test structural models, and interpret complementary experiments.

Although they both adopt stable folds, the TYMV and BMV TLSs are very different in their overall architectures. Both previous experimental results-based models and the new biophysical data presented here show that while the TYMV TLS adopts a shape reminiscent of tRNA, the BMV TLS conformation comprises a dense packing of multiple elements around an RNA four-way junction. This variation in TLS structure parallels trends seen in authentic cellular tRNAs of both cytoplasmic and mitochondrial origin in all kingdoms of life. Specifically, tRNAs vary in the size of variable loops (Helm et al. 2000; Bonnefond et al. 2005), base modifications (Helm 2006), and sequence in homologous tRNAs between different organisms and can be missing entire structural features (such as D or T loops) (Okimoto and Wolstenholme 1990). The functional importance of this variation in authentic tRNAs is not totally understood, but it seems likely that different evolutionary trajectories and different cellular environments have led to this diversity. Diversity in TLSs likely results from the same causes, with the additional need for each to play multiple but differing roles in its respective virus and the hosts it infects. Hence, each TLS has evolved to be a multifunctional 
structured RNA built on a tRNA-like scaffold, but with specific features unique to each RNA.

The TYMV TLS is structurally similar to tRNA, but it is important to place this into context with the details of tRNA structure and dynamics. In cellular tRNAs, a T-stemloop/acceptor stem "minihelix" often behaves as a separate entity that can be aminoacylated and contains the identity elements for recognition by the AARS (Alexander et al. 1998). These data and the presence of mitochondrial tRNAs that lack a D-loop support the idea that minihelices of this type were the evolutionary precursors to modern tRNAs (Schimmel and Ribas de Pouplana 1995). In fact, thermal denaturation of tRNA shows that the D loop and stem unfolds first, and the structure and proper docking of the D loop into the tRNA "elbow" requires the tertiary structure of the rest of the RNA be formed first (Wilkinson et al. 2005). The flexibility of the D loop/stem of tRNA has functional significance; for example the archeaosine tRNAguanine transglycosylase (ArcTGT) disrupts the docking and structure of the D loop/stem while leaving the structure of the T loop/acceptor stem relatively unchanged, inducing formation of "lambda form" tRNA (Ishitani et al. 2003). It is therefore compelling that in the TYMV TLS, the acceptor stem pseudoknot, T loop, and variable arm can structurally organize without the participation of the D loop (Fig. 3). In addition, the D loop of many TLSs related to TYMV are truncated by 1-2 nt, suggesting changes in the way this loop docks into the elbow (de Smit et al. 2002). While we cannot make conclusions regarding the kinetics or order of folding of the TYMV TLS, the relative weakness of the D-loop solvent protection and the fact that it is truncated in many TYMV-related TLSs might show it can "undock" from the elbow while the rest of the molecule remains relatively structurally intact; these local conformational alterations might be important for specific tasks regulated by the TLS. Hence, the TYMV TLS might not only mimic the overall conformation of the tRNA but also some details of how the components of the tRNA thermodynamically, hierarchically, and dynamically relate within the fold.

One conformational change in the TYMV TLS is unfolding of the molecule to allow passage of the RDRP during (-) strand synthesis, which starts at the $3^{\prime}$ end of the viral RNA. Our probing data allow us to speculate and propose a mechanism by which this could occur. The $3^{\prime}$ end acceptor stem pseudoknot appears dynamic and unstable in our probing studies, and the overall tight packing of all elements of the molecule is sensitive to the presence of $5^{\prime}$ sequences that "zipper" the molecule together, affecting all elements of the structure. It therefore seems plausible that binding of the RDRP near the $3^{\prime}$ end of the RNA could readily affect the structure of the pseudoknot, which in turn would affect the interaction of nt 85 , which has been shown to affect Dloop docking, etc. Hence, a disruption of nucleotides 83-85 could lead to a cascading loss of coupled stabilizing in- teractions throughout the TLS, allowing passage of the RDRP. Furthermore, once the polymerase has passed, refolding of the TLS could be readily achieved. Future experiments can test this speculative model.

In contrast to the TYMV TLS, the BMV TLS's conformation diverges substantially from the classic L-shaped structure, perhaps reflecting its multifunctionality. Within the BMV TLS, the acceptor stem pseudoknot has been shown to contain the critical aminoacylation identity elements, thus it has been said that this RNA acts as an aminoacylatable minihelix (Fechter et al. 2001a; Bonnefond et al. 2005). However, footprinting studies with the AARS show contacts in several parts of the RNA outside of the acceptor stem region, including the B2 and C stem-loops (Fechter et al. 2001a), perhaps stabilizing the RNA-protein interaction in a noncanonical way and without an anticodon loop. Our probing confirms these stem loops extend into solution and are available for intermolecular interactions, consistent with the idea that this TLS prefolds into a structure prepared for noncanonical AARS binding. There is precedent for TyrRS interacting with RNAs in divergent ways. Specifically, the neurospora mitochondria TyrRS has been shown to bind to and enhance the activity of a group I intron, using interactions that deviate dramatically from the tRNA-TyrRS interaction. (Guo and Lambowitz 1992; Mohr et al. 1992, 1994; Caprara et al. 1996; Paukstelis et al. 2008). In addition, the TyrRS/tRNA system is unusual and idiosyncratic compared with other aminoacylation systems in several ways, including variations in $\mathrm{tRNA}^{\mathrm{Tyr}}$ structure (particularly in the variable loop), in the domain organization of the TyrRS, in the way the TyrRS and tRNA interact, and with the relative importance and use of identity elements (Bonnefond et al. 2005). Whether or not the idiosyncrasies of the TyrRS/tRNA system relates to the divergent structure of the BMV TLS or not remains an open and interesting question.

The multifunctional nature of the BMV TLS may demand changes in the conformation of the RNA, and as with TYMV, one is unfolding for RDRP passage. A promoter to negative strand BMV RNA synthesis is associated with the $\mathrm{C}$ stem of the BMV TLS and includes a bulge structure (Kim et al. 2000). Increased stability of the helices flanking this bulge by mutation reduces replication efficiency (Kim et al. 2000). Our probing shows both solvent protection and enhancement in this bulge as well as dynamic behavior; thus, this element has parts that are integral to the overall BMV TLS fold, but other parts that are solvent exposed and available for recognition by the RDRP. These observations suggest a model in which initial binding of the RDRP to the exposed portion of this $\mathrm{C}$ bulge induces local unfolding, which partially destabilizes the core fold and reduces the thermodynamic barrier for replication through this region. Hence, analogous to the $5^{\prime}$ nucleotides of the TYMV TLS, disruption of a few interactions within the tertiary structure may be enough to destabilize the entire structure. 
The ability of the TLSs to drive aminoacylation of the $3^{\prime}$ end of viral RNAs naturally led to studies aimed at understanding the structural basis of this function. The data presented here builds on existing knowledge and models regarding TLS structure, helps to establish features of the global architectures of TLS RNAs, and further places them in a biophysical and structural framework that compares them to each other, to tRNAs, and to RNAs in general. Combining these results with previously published data allows new models to be proposed and should help guide future structural and functional studies aimed at developing and testing these and other mechanistic models of these multifunctional RNAs.

\section{MATERIALS AND METHODS}

\section{DNA/Plasmid construction}

DNA constructs containing, from the $5^{\prime}$ end, an EcoRI restriction site, the T7 RNA polymerase promoter, a hammerhead ribozyme, the RNA sequence of interest, the hepatitis delta virus ribozyme (HDV $\mathrm{Rz}$ ), and a BamH1 restriction site were created using overlapping primer PCR with $P f u$ polymerase. The resulting DNA fragment either was ligated into pUC19, propagated in Eschrichia coli, and verified by sequencing or used directly as templates for transcription.

\section{RNA transcription and purification}

RNA was transcribed in vitro from DNA templates with T7 RNA polymerase and purified by denaturing gel electrophoresis as described (Kieft et al. 2007). Templates for transcription were either PCR products or BamH1 linearized plasmids. RNAs used in SHAPE experiments were transcribed from PCR-created DNA templates produced using the plasmids described above and M13 forward primer and a primer containing the sequence $5^{\prime}$ CCAGCGAGGAGGCTGGGACCATGC-3' (“internal delta primer”) that lies just $3^{\prime}$ of the TLS sequence in the HDV Rz.

\section{Radiolabeling of DNA and RNA}

RNA and DNA were $5^{\prime}$-end labeled as described (Kieft et al. 2007).

\section{Hydroxyl radical probing}

Hydroxyl radical probing was performed and analyzed essentially as described, as was generation of RNase T1 and hydrolysis ladders (Kieft et al. 2007). RNA was heated in DEPC-treated water for $1 \mathrm{~min}$ at $85^{\circ} \mathrm{C}$ and cooled for $20 \mathrm{~min}$ at room temperature, then placed in $30 \mathrm{mM}$ HEPES-NaOH ( $\mathrm{pH} 7.5$ ), 0 or $10 \mathrm{mM} \mathrm{MgCl}_{2}$, and $0.2 \mathrm{mg} / \mathrm{mL}$ total yeast tRNA. The reaction incubated for 10 min at $30^{\circ} \mathrm{C}$, and then cleavage reactions were conducted for 2 min at $30^{\circ} \mathrm{C}$, resolved on $10 \%$ sequencing denaturing polyacrylamide gels ( $1 \times \mathrm{TBE}, 7 \mathrm{M}$ urea, 29:1 acrylamide:bis-acrylamide). Gels were dried and visualized with a phosphorimager.

\section{Selective 2' hydroxyl acylation analyzed by primer extension.}

SHAPE was performed as essentially described (Merino et al. 2005) with modification: 4 pmol RNA was heated for $1 \mathrm{~min}$ at $85^{\circ} \mathrm{C}$, cooled for $20 \mathrm{~min}$ at room temperature, then placed in
$100 \mathrm{mM}$ HEPES-KOH (pH 7.5), $100 \mathrm{mM} \mathrm{NaCl}$, and $7 \mathrm{mM} \mathrm{MgCl}$; $10 \mu \mathrm{L}$ reactions with $\mathrm{N}$-methylisatoic anhydride (NMIA) were conducted for $1 \mathrm{~h}$ at $35^{\circ} \mathrm{C}$ and desalted with a Bio-Rad Micro Bio-Spin P-30 column. Reverse transcription reactions were completed and resolved by gel electrophoresis as in hydroxyl radical probing. ImageQuant and Excel were used to normalize and analyze the data. Modifications that were visually apparent but between $10 \%$ and $25 \%$ of intensity of the strongest modification band in the gel were considered weak, modifications above $25 \%$ were considered strong.

\section{Analytical ultracentrifugation.}

Conducted similarly to previous studies (Costantino and Kieft 2005; Kieft et al. 2007). Briefly, RNA was heated for 1 min to $85^{\circ} \mathrm{C}$, cooled to room temperature, then placed in $50 \mathrm{mM} \mathrm{Na}$ Cacodylate (pH 6.5), 0 or $10 \mathrm{mM} \mathrm{MgCl}_{2}$. Final [RNA] was $\sim 0.08$ $\mathrm{mg} / \mathrm{mL}$. SV/AUC was performed in a Beckman XLA centrifuge using an 8-position An50Ti rotor. Samples were spun at 27K rpm for the TYMV TLS, A. thaliana tRNA ${ }^{\text {Val }}$, and the TMV TLS, and $30 \mathrm{~K}$ for the BMV TLS. Scans were collected every $7 \mathrm{~min}$ at $280 \mathrm{~nm}$, with a total of at least 45 scans per sample. A minimum of 35 scans were analyzed as dc/R using SVEDBERG V6.39 (Philo 1997). SEDNTRP then was used to calculate $R_{H}$ and $f / f_{0}$, using specific volume of $0.53 \mathrm{~cm}^{3} / \mathrm{g}$.

\section{Small-angle X-ray scattering}

RNA was heated for $1 \mathrm{~min}$ at $85^{\circ} \mathrm{C}$, cooled for $10 \mathrm{~min}$ at room temperature, then diluted in 50 -fold gel-filtration buffer $(20 \mathrm{mM}$ MOPS at $\mathrm{pH} 6.5,50 \mathrm{mM} \mathrm{KCl}$, and either $7.6 \mathrm{mM} \mathrm{MgCl}_{2}$ or $1 \mathrm{mM}$ EDTA), concentrated to $20 \mu \mathrm{L}$, and run through a Superose $6 \mathrm{PC}$ 3.2 column on an Ettan LC liquid chromatography system (GE Healthcare) configured with an in-line vacuum degasser (Rheodyne) and a $0.01 \mu \mathrm{m}$ solvent filter (Millipore). Elution was monitored by a light scattering system composed of an 18-angle DAWN HELEOS detector, an Optilab refractive index concentration detector, and a DynaPro quasi-elastic light scattering detector (Wyatt Technology). Following this analytical scale chromatography, preparative scale samples were prepared with a final injection concentration of $\sim 10 \mathrm{mg} / \mathrm{mL}$ in $50 \mu \mathrm{L}$. Peaks corresponding to monodisperse RNA sample were immediately used for SAXS at beamline 12.3.1 of the Advanced Light Source using $20 \mu \mathrm{L}$ samples loaded from a 96-well plate using a Hamilton syringe robot. Exposures of 5, 50, and 5 sec were collecte,d and the two 5 -sec exposures were compared to check for radiation damage. Matching buffer was exposed identically and subtracted to generate the macromolecule's SAXS profile. Each sample was checked for interparticle interference by collecting SAXS profiles as a concentration range (two-thirds dilution series). In all cases, no significant interparticle interference was observed as indicated by the superimposition of the scattered intensities at the lowest scattering angles. Exposures (at 5 and $50 \mathrm{sec}$ ) were merged into a single scattering profile using the program Primus, then Gnom was used to estimate $\mathrm{D}_{\max }$ and to generate output for $a b$ initio shape reconstruction analysis by Gasbor.

\section{ACKNOWLEDGMENTS}

We thank David Barton, Robert Batey, David Costantino, and Theo Dreher for critical reading of this manuscript and for many 
useful suggestions, and to all members of the Kieft Laboratory for useful discussions. Files containing the coordinates of the BMV and TYMV TLS models were a kind gift of the TLS group in the laboratory of Richard Giegé. This work was supported by NIH grant R03AI072187 to J.S.K.

Received September 11, 2008; accepted November 3, 2008.

\section{REFERENCES}

Alexander, R.W., Nordin, B.E., and Schimmel, P. 1998. Activation of microhelix charging by localized helix destabilization. Proc. Natl. Acad. Sci. 95: 12214-12219.

Annamalai, P. and Rao, A.L. 2007. In vivo packaging of brome mosaic virus RNA 3, but not RNAs 1 and 2, is dependent on a cis-acting $3^{\prime}$ tRNA-like structure. J. Virol. 81: 173-181.

Barends, S., Bink, H.H., van den Worm, S.H., Pleij, C.W., and Kraal, B. 2003. Entrapping ribosomes for viral translation: tRNA mimicry as a molecular Trojan horse. Cell 112: 123-129.

Bonnefond, L., Giege, R., and Rudinger-Thirion, J. 2005. Evolution of the tRNA ${ }^{\text {Tyr }} /$ TyrRS aminoacylation systems. Biochimie 87: 873-883.

Caprara, M.G., Mohr, G., and Lambowitz, A.M. 1996. A tyrosyl-tRNA synthetase protein induces tertiary folding of the group I intron catalytic core. J. Mol. Biol. 257: 512-531.

Costantino, D. and Kieft, J.S. 2005. A preformed compact ribosomebinding domain in the cricket paralysis-like virus IRES RNAs. RNA 11: 332-343.

de Smit, M.H., Gultyaev, A.P., Hilge, M., Bink, H.H., Barends, S., Kraal, B., and Pleij, C.W. 2002. Structural variation and functional importance of a D-loop-T-loop interaction in valine-accepting tRNA-like structures of plant viral RNAs. Nucleic Acids Res. 30: 4232-4240.

Dreher, T.W. 2008. Role of tRNA-like structures in controlling plant virus replication. Virus Res. (in press). doi: 10.1016/j.virusres. 2008.06.010.

Dreher, T.W., Bujarski, J.J., and Hall, T.C. 1984. Mutant viral RNAs synthesized in vitro show altered aminoacylation and replicase template activities. Nature 311: 171-175.

Dreher, T.W., Florentz, C., and Giege, R. 1988. Valylation of tRNAlike transcripts from cloned cDNA of turnip yellow mosaic virus RNA demonstrate that the L-shaped region at the $3^{\prime}$ end of the viral RNA is not sufficient for optimal aminoacylation. Biochimie 70: $1719-1727$.

Dumas, P., Moras, D., Florentz, C., Giege, R., Verlaan, P., Van Belkum, A., and Pleij, C.W. 1987. 3-D graphics modelling of the tRNA-like $3^{\prime}$-end of turnip yellow mosaic virus RNA: Structural and functional implications. J. Biomol. Struct. Dyn. 4: 707-728.

Fechter, P., Giege, R., and Rudinger-Thirion, J. 2001a. Specific tyrosylation of the bulky tRNA-like structure of brome mosaic virus RNA relies solely on identity nucleotides present in its amino acid-accepting domain. J. Mol. Biol. 309: 387-399.

Fechter, P., Rudinger-Thirion, J., Florentz, C., and Giege, R. 2001b. Novel features in the tRNA-like world of plant viral RNAs. Cell. Mol. Life Sci. 58: 1547-1561.

Felden, B., Florentz, C., Giege, R., and Westhof, E. 1994. Solution structure of the $3^{\prime}$-end of brome mosaic virus genomic RNAs. Conformational mimicry with canonical tRNAs. J. Mol. Biol. 235: 508-531.

Felden, B., Florentz, C., Giege, R., and Westhof, E. 1996. A central pseudoknotted three-way junction imposes tRNA-like mimicry and the orientation of three $5^{\prime}$ upstream pseudoknots in the $3^{\prime}$ terminus of tobacco mosaic virus RNA. RNA 2: 201-212.

Friederich, M.W., Vacano, E., and Hagerman, P.J. 1998. Global flexibility of tertiary structure in RNA: Yeast tRNA ${ }^{\text {Phe }}$ as a model system. Proc. Natl. Acad. Sci. 95: 3572-3577.
Garcia-Arenal, F. 1988. Sequence and structure at the genome 3' end of the U2-strain of tobacco mosaic virus, a histidine-accepting tobamovirus. Virology 167: 201-206.

Giege, R., Rudinger, J., Dreher, T., Perret, V., Westhof, E., Florentz, C., and Ebel, J.P. 1990. Search of essential parameters for the aminoacylation of viral tRNA-like molecules. Comparison with canonical transfer RNAs. Biochim. Biophys. Acta 1050: 179185.

Grundy, F.J. and Henkin, T.M. 1993. tRNA as a positive regulator of transcription antitermination in B. subtilis. Cell 74: 475-482.

Guo, Q. and Lambowitz, A.M. 1992. A tyrosyl-tRNA synthetase binds specifically to the group I intron catalytic core. Genes \& Dev. 6: 1357-1372.

Hall, T.C., Shih, D.S., and Kaesberg, P. 1972. Enzyme-mediated binding of tyrosine to brome-mosaic-virus ribonucleic acid. Biochem. J. 129: 969-976.

Helm, M. 2006. Post-transcriptional nucleotide modification and alternative folding of RNA. Nucleic Acids Res. 34: 721-733.

Helm, M., Brule, H., Friede, D., Giege, R., Putz, D., and Florentz, C. 2000. Search for characteristic structural features of mammalian mitochondrial tRNAs. RNA 6: 1356-1379.

Ishitani, R., Nureki, O., Nameki, N., Okada, N., Nishimura, S., and Yokoyama, S. 2003. Alternative tertiary structure of tRNA for recognition by a posttranscriptional modification enzyme. Cell 113: 383-394.

Joshi, S., Chapeville, F., and Haenni, A.L. 1982. Length requirements for tRNA-specific enzymes and cleavage specificity at the $3^{\prime}$ end of turnip yellow mosaic virus RNA. Nucleic Acids Res. 10: 19471962.

Joshi, R.L., Joshi, S., Chapeville, F., and Haenni, A.L. 1983. tRNA-like structures of plant viral RNAs: Conformational requirements for adenylation and aminoacylation. EMBO J. 2: 1123-1127.

Joshi, R.L., Chapeville, F., and Haenni, A.L. 1985. Conformational requirements of tobacco mosaic virus RNA for aminoacylation and adenylation. Nucleic Acids Res. 13: 347-354.

Kieft, J.S., Costantino, D.A., Filbin, M.E., Hammond, J., and Pfingsten, J.S. 2007. Structural methods for studying IRES function. Methods Enzymol. 430: 333-371.

Kim, C.H., Kao, C.C., and Tinoco Jr., I. 2000. RNA motifs that determine specificity between a viral replicase and its promoter. Nat. Struct. Biol. 7: 415-423.

Kolk, M.H., van der Graaf, M., Wijmenga, S.S., Pleij, C.W., Heus, H.A., and Hilbers, C.W. 1998. NMR structure of a classical pseudoknot: Interplay of single- and double-stranded RNA. Science 280: 434-438.

Latham, J.A. and Cech, T.R. 1989. Defining the inside and outside of a catalytic RNA molecule. Science 245: 276-282.

Mans, R.M., Verlaan, P.W., Pleij, C.W., and Bosch, L. 1990. Aminoacylation of $3^{\prime}$ terminal tRNA-like fragments of turnip yellow mosaic virus RNA: the influence of $5^{\prime}$ nonviral sequences. Biochim. Biophys. Acta 1050: 186-192.

Matsuda, D. and Dreher, T.W. 2007. Cap- and initiator tRNAdependent initiation of TYMV polyprotein synthesis by ribosomes: Evaluation of the Trojan horse model for TYMV RNA translation. RNA 13: 129-137.

Merino, E.J., Wilkinson, K.A., Coughlan, J.L., and Weeks, K.M. 2005. RNA structure analysis at single nucleotide resolution by selective 2 '-hydroxyl acylation and primer extension (SHAPE). J. Am. Chem. Soc. 127: 4223-4231.

Mohr, G., Zhang, A., Gianelos, J.A., Belfort, M., and Lambowitz, A.M. 1992. The neurospora CYT-18 protein suppresses defects in the phage T4 td intron by stabilizing the catalytically active structure of the intron core. Cell 69: 483-494.

Mohr, G., Caprara, M.G., Guo, Q., and Lambowitz, A.M. 1994. A tyrosyl-tRNA synthetase can function similarly to an RNA structure in the Tetrahymena ribozyme. Nature 370: 147150 . 
Okimoto, R. and Wolstenholme, D.R. 1990. A set of tRNAs that lack either the $\mathrm{T} \psi \mathrm{C}$ arm or the dihydrouridine arm: Toward a minimal tRNA adaptor. EMBO J. 9: 3405-3411.

Osman, T.A., Hemenway, C.L., and Buck, K.W. 2000. Role of the 3' tRNA-like structure in tobacco mosaic virus minus-strand RNA synthesis by the viral RNA-dependent RNA polymerase in vitro. $J$. Virol. 74: 11671-11680.

Paukstelis, P.J., Chen, J.H., Chase, E., Lambowitz, A.M., and Golden, B.L. 2008. Structure of a tyrosyl-tRNA synthetase splicing factor bound to a group I intron RNA. Nature 451: 94-97.

Philo, J.S. 1997. An improved function for fitting sedimentation velocity data for low-molecular-weight solutes. Biophys. J. 72: 435444.

Pinck, M., Yot, P., Chapeville, F., and Duranton, H.M. 1970. Enzymatic binding of valine to the $3^{\prime}$ end of TYMV-RNA. Nature 226: 954-956.

Rietveld, K., Van Poelgeest, R., Pleij, C.W., Van Boom, J.H., and Bosch, L. 1982. The tRNA-like structure at the $3^{\prime}$ terminus of turnip yellow mosaic virus RNA. Differences and similarities with canonical tRNA. Nucleic Acids Res. 10: 1929-1946.

Rietveld, K., Pleij, C.W., and Bosch, L. 1983. Three-dimensional models of the tRNA-like $3^{\prime}$ termini of some plant viral RNAs. EMBO J. 2: 1079-1085.

Rietveld, K., Linschooten, K., Pleij, C.W., and Bosch, L. 1984. The three-dimensional folding of the tRNA-like structure of tobacco mosaic virus RNA. A new building principle applied twice. EMBO J. 3: $2613-2619$.

Robertus, J.D., Ladner, J.E., Finch, J.T., Rhodes, D., Brown, R.S., Clark, B.F., and Klug, A. 1974. Structure of yeast phenylalanine tRNA at $3 \AA$ resolution. Nature 250: 546-551.

Schimmel, P. and Ribas de Pouplana, L. 1995. Transfer RNA: From minihelix to genetic code. Cell 81: 983-986.
Sela, I. 1972. Tobacco enzyme-cleaved fragments of TMV-RNA specifically accepting serine and methionine. Virology 49: 90-94.

Sheppard, K., Yuan, J., Hohn, M.J., Jester, B., Devine, K.M., and Soll, D. 2008. From one amino acid to another: tRNA-dependent amino acid biosynthesis. Nucleic Acids Res. 36: 1813-1825.

Shi, H. and Moore, P.B. 2000. The crystal structure of yeast phenylalanine tRNA at $1.93 \AA$ resolution: A classic structure revisited. RNA 6: 1091-1105.

Singh, R.N. and Dreher, T.W. 1997. Turnip yellow mosaic virus RNAdependent RNA polymerase: Initiation of minus strand synthesis in vitro. Virology 233: 430-439.

Suddath, F.L., Quigley, G.J., McPherson, A., Sneden, D., Kim, J.J., Kim, S.H., and Rich, A. 1974. Three-dimensional structure of yeast phenylalanine transfer RNA at $3.0 \AA$ resolution. Nature 248: 20-24.

Svergun, D.I., Petoukhov, M.V., and Koch, M.H. 2001. Determination of domain structure of proteins from X-ray solution scattering. Biophys. J. 80: 2946-2953.

Tsonis, P.A. and Dwivedi, B. 2008. Molecular mimicry: Structural camouflage of proteins and nucleic acids. Biochim. Biophys. Acta 1783: $177-187$.

Wain-Hobson, S., Sonigo, P., Danos, O., Cole, S., and Alizon, M. 1985. Nucleotide sequence of the AIDS virus, LAV. Cell 40: 9-17.

Wilkinson, K.A., Merino, E.J., and Weeks, K.M. 2005. RNA SHAPE chemistry reveals nonhierarchical interactions dominate equilibrium structural transitions in $\mathrm{tRNA}^{\mathrm{Asp}}$ transcripts. J. Am. Chem. Soc. 127: 4659-4667.

Zeenko, V.V., Ryabova, L.A., Spirin, A.S., Rothnie, H.M., Hess, D., Browning, K.S., and Hohn, T. 2002. Eukaryotic elongation factor $1 \mathrm{~A}$ interacts with the upstream pseudoknot domain in the $3^{\prime}$ untranslated region of tobacco mosaic virus RNA. J. Virol. 76: 5678-5691. 

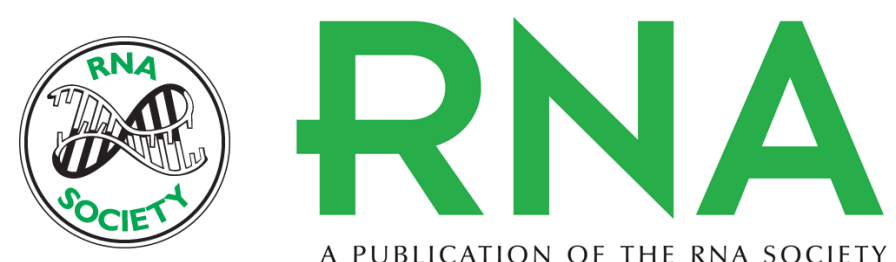

A PUBLICATION OF THE RNA SOCIETY

\section{Comparison and functional implications of the 3D architectures of viral tRNA-like structures}

John A. Hammond, Robert P. Rambo, Megan E. Filbin, et al.

RNA 2009 15: 294-307

References This article cites 55 articles, 14 of which can be accessed free at:

http://rnajournal.cshlp.org/content/15/2/294.full.html\#ref-list-1

\section{License}

Email Alerting Receive free email alerts when new articles cite this article - sign up in the box at the Service top right corner of the article or click here. 